Pacific Journal of Mathematic 


\title{
RELATIVIZATION AND EXTENSION OF SOLUTIONS OF IRREFLEXIVE RELATIONS
}

\author{
MOSES RICHARDSON
}

1. Introduction. Let $\succ$ be an irreflexive binary relation defined over a domain $\mathcal{D}$ of elements $a, b, c, \ldots$ We represent the system $(\mathcal{O},>)$ by an oriented graph $G$ by regarding the elements of $\mathcal{S}$ as vertices of $G$ and inserting an arc $a b$ of the graph, oriented from $a$ to $b$, if and only if $a \succ b$. The sentence " $a \succ b$ ", is read " $a$ dominates $b$ ". A set $V$ of vertices is termed internally satisfactory" if and only if $x \in V$ and $y \in V$ implies $x \ngtr y$. A set $V$ of vertices is termed $e x$ ternally satisfactory if and only if $y \in \mathscr{D}-V$ implies that there exists an $x \in V$ such that $x>y$. A set $V$ of vertices is termed a solution of $G$, or of $(\mathscr{D}, \succ)$, if and only if it is both internally and externally satisfactory. In [4], various sufficient conditions for the existence of solutions were established.

By a subsystem $\left(\mathscr{D}_{0}, \succ\right)$ of the system $(\mathscr{D}, \succ)$ is meant a system where $D_{0} \subset D$ and the relation $\succ$ for the subsystem is merely the restriction of the relation $\succ$ for the supersystem $(\mathscr{D}, \succ)$. Let $G_{0}$ be the graph of the subsystem ( () $0, \succ$ ) and let $V_{0}$ be a solution of $G_{0}$. A solution $V$ of $G$ is termed an extension of $V_{0}$ if $V \cap \mathscr{S}_{0}=V_{0}$; in this case $V_{0}$ is also said to be relativized from $V$. In this paper, some sufficient conditions for the existence of relativizations and extensions of solutions are presented. More elegant and more effective extension theorems, especially with a view toward possible applications to the theory of $n$-person games, remain to be desired. It is hoped that the present paper may serve to stimulate interest in this apparently difficult problem.

2. A theorem on relativization. If $H$ is a subgraph of the graph $G$, then the graph obtained by adding to $H$ all the arcs of $G$ which join pairs of vertices of $H$ will be termed the juncture of $H$ (relative to $G$ ) and will be denoted by $\bar{H}$.

${ }^{1}$ In [2], internally satisfactory is called satisfactory with respect to non-domination, and in $[4]$ it is called $*$-satisfactory.

Received March 1, 1954. Part of the work of this paper was done at the Institute for Advanced Study in 1952-3, and part while the author was consultant to the Logistics Project sponsored by the Office of Naval Research in the Department of Mathematics at Princeton University in 1953-4. A statement of many of the results contained herein appeared without proofs in [5].

Pacific J. Math. 5 (1955), 551-584 
$H$ is termed a conjunct subgraph of $G$ if and only if $H=\bar{H}$.

The graph $G_{0}$ of a subsystem $\left(\vartheta_{0}, \succ\right)$ of the system $(\mathscr{D}, \succ)$ having the graph $G$ is a conjunct closed subgraph of $G$. If $H$ is any subgraph of $G$, proper or not, and $x$ is any vertex of $G$, then $D^{-1}(x, H)$ shall denote the set of all vertices $y$ of $H$ such that $y \succ x$. If $X$ is any set of vertices of $G$, let

$$
D^{-1}(X, H)=\bigcup_{x \in X} D^{-1}(x, H),
$$

and let

$$
D^{-n}(X, H)=D^{-1}\left(D^{-n+1}(X, H), H\right)
$$

for $n>1$. Let $D^{\circ}(X, H)=X$ by definition.

THEOREM 1. If $G_{0}$ is a conjunct subgraph of $G$ and $V$ is a solution of $G$, then a sufficient condition for $V \cap \oslash_{0}$ to be a solution of $G_{0}$, where $\bigoplus_{0}$ is the set of vertices of $G_{0}$, is that

$$
D^{-1}(y, G) \subset \mathscr{D}_{0} \quad \text { for every } y \in \mathscr{D}_{0}-V \cap \mathscr{D}_{0}
$$

Proof. We must prove that $V \cap \mathscr{D}_{0}$ is both internally and externally satisfactory with respect to $G_{0}$. That is we must prove that

(a) $x, y \in V \cap \mathscr{D}_{0}$ implies $x \ngtr y$ relative to $G_{0}$, and

(b) $y \in \mathscr{D}_{0}-V \cap \mathscr{D}_{0}$ implies that there exists an $x \in V \cap \mathscr{D}_{0}$ such that $x>y$ relative to $G_{0}$.

But (a) follows immediately from the facts that $G_{0}$ is a conjunct subgraph of $G$ and that $V$ is a solution of $G$. To prove (b), consider any $y \in \mathscr{I}_{0}-V \cap \mathscr{D}_{0}$. There exists an $x \in V$ such that $x>y$ relative to $G$ since $V$ is a solution of $G$. Then $x \in D^{-1}(y, G) \subset \mathscr{I}_{0}$ by hypothesis. Thus $x, y \in \mathscr{I}_{0}$ and the oriented arc $x y \subset G$. Since $G_{0}$ is a conjunct subgraph of $G$, arc $x y \subset G_{0}$. This completes the proof.

REMARK. It would suffice to replace Condition $(1)$ by the weaker condition: $y \in \mathscr{D}_{0}-V \cap \mathscr{D}_{0}$ implies that there exists a vertex $x \in V \cap \mathscr{D}_{0}$ such that $x>y$.

3. An extension theorem. If $X \subset \mathscr{D}$, let the predecessor-set of $X$ relative to $G-G_{0}$ denote the set 


$$
P\left(X, G-G_{0}\right)=\bigcup_{n=1}^{\infty} D^{-n}\left(X, G-G_{0}\right)
$$

By a predecessor-sequence $p\left(x_{0}, G-G_{0}\right)$ of $x_{0} \in \mathscr{O}_{0}$ relative to $G-G_{0}$ is meant a maximal regression ${ }^{2} x_{0}, x_{1}, x_{2}, \ldots$, of finite or infinite length, such that all its vertices except possibly $x_{0}$ itself are in $G-G_{0}$; that is, such that one vertex $x_{n}$ is chosen from the set $D^{-1}\left(x_{n-1}, G-G_{0}\right)$ for each $n>0$, all $x_{n}$ 's being distinct. Let $p^{*}\left(x_{0}, G-G_{0}\right)$ be the set of all vertices of the predecessor-sequence $p\left(x_{0}, G-G_{0}\right)$ other than $x_{0}$ itself. A predecessor-sequence is termed trivial if and only if $p^{*}\left(x_{0}, G-G_{0}\right)$ is empty. We have

$$
P\left(x_{0}, G-G_{0}\right)=U_{p} *\left(x_{0}, G-G_{0}\right)
$$

for all predecessor-sequences $p\left(x_{0}, G-G_{0}\right)$ of $x_{0}$ relative to $G-G_{0}$. Note that the elements of the predecessor-set of $x_{0}$ or of a predecessor-sequence of $x_{0}$ are not necessarily ancestors of $x_{0}$, although every ancestor of $x_{0}$ belongs to at least one predecessor-sequence of $x_{0}$ (all relative to $G-G_{0}$ ). If $>$ is not asymmetric then a source, which has no ancestor, may have non-trivial predecessor-sequences.

Throughout the sequel we suppose that $G_{0}$ is the graph of a subsystem $\left(\mathcal{D}_{0}, \succ\right.$ ) of the system $\left(D, \succ\right.$ ) the graph of which is $G$, that $V_{0}$ is a given solution of $G_{0}$, and that ${ }^{3}$

$$
W_{00}=D\left(V_{0}, G_{0}\right)=\mathscr{D}_{0}-V_{0}
$$

THEOREM 2. Suppose that:

(1) All non-trivial predecessor-sequences $p\left(x_{0}, G-G_{0}\right), x_{0} \in \mathscr{D}_{0}$, are either infinite or, if finite, of odd length if $x_{0} \in W_{00}$ ana' of even length if $x_{0} \in V_{0}$;

(2) $D\left(V_{0}, G\right) \cap D^{-2 n}\left(V_{0}, G-G_{0}\right)=D\left(V_{0}, G\right) \cap D^{-2 n+1}\left(\mathbb{W}_{00}, G-G_{0}\right)=0$ for all $n>0$;

(3) If $h>0$ and $k>0$ are of the same parity then

$$
D^{-h}\left(V_{0}, G-G_{0}\right) \cap D^{-k}\left(W_{00}, G-G_{0}\right)=0 \text {, }
$$

and if $h>0$ and $k>0$ are of different parities then

$D^{-h}\left(V_{0}, G-G_{0}\right) \cap D^{-k}\left(V_{0}, G-G_{0}\right)=D^{-h}\left(\mathbb{W}_{00}, G-G_{0}\right) \cap D^{-k}\left(\mathbb{W}_{00}, G-G_{0}\right)=0 ;$

2See [4] for definitions omitted here.

3 This is a slight modification of the notation of [4]. 
(4) $\mathscr{D}-\mathscr{D}_{0} \subset P\left(\mathscr{D}_{0}, G-G_{0}\right)$.

Then a solution $V$ of $G$ which is an extension of $V_{0}$ exists.

Proof. Let

$$
\begin{aligned}
& V=V_{0} \cup\left(\bigcup_{n=1}^{\infty} D^{-2 n}\left(V_{0}, G-G_{0}\right)\right) \cup\left(\bigcup_{n=1}^{\infty} D^{-2 n+1}\left(W_{00}, G-G_{0}\right)\right), \\
& W=W_{00} \cup\left(\bigcup_{n=1}^{\infty} D^{-2 n+1}\left(V_{0}, G-G_{0}\right)\right) \cup\left(\bigcup_{n=1}^{\infty} D^{-2 n}\left(W_{00}, G-G_{0}\right)\right) .
\end{aligned}
$$

We shall show that $V$ is a solution of $G$. Since $G_{0}$ is a conjunct subgraph of $G$ and $V_{0}$ is a solution of $G_{0}$, it follows that $V_{0}$ is internally satisfactory relative to $G$. By (4), $\supseteq=V \cup W$. By (3), $V \cap W^{\prime}=0$; hence $W=\supseteq-V$. We have only to prove:

$$
V \cap D(V, G)=0
$$

$$
W \subset D(V, G) \text {. }
$$

Proof of (a). If $x \in V_{0}, y \in V_{0}$, then $x \ngtr y$ since $V_{0}$ is internally satisfactory relative to $G$.

If $x \in V_{0}, y \in D^{-2 n}\left(V_{0}, G-G_{0}\right)$, then $x \ngtr y$ by $(2)$.

If $x \in V_{0}, y \in D^{-2 n+1}\left(W_{00}, G-G_{0}\right)$, then $x \ngtr y$ by $(2)$.

If $x \in D^{-2 n}\left(V_{0,} G-G_{0}\right), y \in V_{0}$, then $x \nsucc y$; for $x \succ y$ would imply that $x \in D^{-1}\left(V_{0}, G-G_{0}\right)$ contrary to (3).

If $x \in D^{-2 n}\left(V_{0}, G-G_{0}\right), y \in D^{-2 m}\left(V_{0}, G-G_{0}\right)$, then $x \ngtr y$; for $x \succ y$ would imply that $x \in D^{-2 m-1}\left(V_{0}, G-G_{0}\right)$ contrary to (3).

If $x \in D^{-2 n}\left(V_{0}, G-G_{0}\right), y \in D^{-2 m+1}\left(W_{00}, G-G_{0}\right)$, then $x \ngtr y$; for $x \succ y$ would imply that $x \in D^{-2 m}\left(\mathbb{W}_{00}, G-G_{0}\right)$ contrary to (3).

If $x \in D^{-2 m+1}\left(\mathbb{W}_{00}, G-G_{0}\right), y \in V_{0}$, then $x \nsucc y$; for $x \succ y$ would imply that $x \in D^{-1}\left(V_{0}, G-G_{0}\right)$, contrary to (3).

If $x \in D^{-2 m+1}\left(\mathbb{W}_{00}, G-G_{0}\right), y \in D^{-2 n}\left(V_{0}, G-G_{0}\right)$, then $x \ngtr y$; for $x \succ y$ would imply that $x \in D^{-2 n-1}\left(V_{0}, G-G_{0}\right)$ contrary to (3). 
If $x \in D^{-2 m+1}\left(W_{00}, G-G_{0}\right), y \in D^{-2 n+1}\left(W_{00}, G-G_{0}\right)$, then $x \nsucc y$; for $x>y$ would imply that $x \in D^{-2 n}\left(\mathbb{W}_{00}, G-G_{0}\right)$ contrary to (3).

Proof of (b). If $y \in \mathbb{W}_{00}$, then there exists an $x \in V_{0}$ such that $x>y$.

If $y \in D^{-2 n+1}\left(V_{0}, G-G_{0}\right)$, then there exists an $x \in D^{-2 n}\left(V_{0}, G-G_{0}\right)$ such that $x>y$, since $y$ belongs to some predecessor-sequence $p\left(x_{0}, G-G_{0}\right)$ of some $x_{0} \in V_{0}$ and such a predecessor-sequence is infinite or of even length by (1).

If $y \in D^{-2 n}\left(\mathbb{W}_{00}, G-G_{0}\right)$, then there exists an $x \in D^{-2 n-1}\left(W_{00}, G-G_{0}\right)$ such that $x \succ y$, since $y$ belongs to some predecessor-sequence $p\left(x_{0}, G-G_{0}\right)$ of some $x_{0} \in \mathbb{W}_{00}$, and such a predecessor-sequence is infinite or of odd length by (1). This completes the proof.

Corollary. Suppose Conditions (1) and (4) of the theorem above, and that:

(a) No vertex of any $P\left(x_{0}, G+G_{0}\right), x_{0} \in \mathfrak{D}_{0}$, is adjacent to any vertex of $\mathscr{\vartheta}_{0}$ other than $x_{0}$; and if $x_{0}$ and $x_{0}^{\prime}$ are distinct vertices of $\mathscr{D}_{0}$ then

$$
P\left(x_{0}, G-G_{0}\right) \cap P\left(x_{0}^{\prime}, G-G_{0}\right)=0 ;
$$

(b) No $P\left(x_{0}, G-G_{0}\right) \cup\left(x_{0}\right), x_{0} \in \mathscr{D}_{0}$, contains an odd unoriented cycle.

Then a solution $V$ of $G$ which is an extension of $V_{0}$ exists.

Proof. We have to show that the hypotheses of the corollary imply those of the theorem. It will suffice to show that if either (2) or (3) are false then either ( $a$ ) or ( $b$ ) will be violated.

If ( 2 ) were false, there would exist either a vertex

$$
x \in D\left(V_{0}, G\right) \cap D^{-2 n}\left(V_{0}, G-G_{0}\right)
$$

or a vertex

$$
y \in D\left(V_{0}, G\right) \cap D^{-2 n+1}\left(W_{00}, G-G_{0}\right) .
$$

In either case, the first part of (a) or (b) is contradicted.

If ( 3 ) were false there would exist either

(i) a vertex 


$$
x \in D^{-h}\left(v_{0}^{i}, G-G_{0}\right) \cap D^{-k}\left(w_{00}^{j}, G-G_{0}\right)
$$

with $h$ and $k$ of the same parity or

(ii) a vertex $y$ such that either

$$
y \in D^{-h}\left(v_{0}^{i}, G-G_{0}\right) \cap D^{-k}\left(v_{0}^{j}, G-G_{0}\right)
$$

or

$$
y \in D^{-h}\left(w_{00}^{i}, G-G_{0}\right) \cap D^{-k}\left(w_{00}^{j}, G-G_{0}\right)
$$

with $h$ and $k$ of different parities.

In Case (i), Condition (a) would be violated. In Case (ii), (a) implies $i=j$. But then $P\left(v_{0}^{i}, G-G_{0}\right) \cup\left(v_{0}^{i}\right)$ or $P\left(w_{00}^{i}, G-G_{0}\right) \cup\left(w_{00}^{i}\right)$ would contain an unoriented cycle of odd length $h+k$ contrary to $(\mathrm{b})$.

4. Sinks and inverse bases. We suppose henceforth that $\mathscr{D}-\mathscr{D}_{0} \subset P\left(\mathscr{D}_{0}\right.$, $\left.G-G_{0}\right)$. If $H$ is any conjunct subgraph of $G$, and $x$ is a vertex of $G$, let

$$
C^{-1}(x, H)=\bigcup_{n=0}^{\infty} D^{-n}(x, H) .
$$

That is, $C^{-1}(x, H)$ denotes the set of all vertices $y$ of $H$ which chain-dominate $x$ by means of a chain all the vertices of which, except possibly $x$, lie in $H$, together with $x$ itself; in symbols

$$
C^{-1}(x, H)=P(x, H) \cup(x) .
$$

If $y \in C^{-1}(x, H)$ and $x \in C^{-1}(y, H), x \neq y$, then $x$ and $y$ are termed cyclically related relative to $H$. If $y \in C^{-1}(x, H)$ but $x \notin C^{-1}(y, H)$ then $x$ is termed a descendant of $y$ relative to $H$. A sequence $x_{1}, x_{2}, x_{3}, \ldots$ of vertices of $H$ is termed a descending sequence of $H$ if $x_{n+1}$ is a descendant of $x_{n}$ for all $n$ (except the last $n$ if the sequence is finite) and if there exists no vertex $y$ which is a descendant of all $x_{n}$. If a vertex $x$ of $H$ has no descendant relative to $H$ then $C^{-1}(x, H)$ is termed an inverse basic set of $H$ and $x$ is termed a sink of this inverse basic set. A subgraph $H$ is termed descendingly finite if every descending sequence of $H$ is finite. The same inverse basic set may contain more than one sink; all sinks of the same inverse basic set are cyclically 
related relative to $H$, and any vertex cyclically related to a sink is a sink of the same inverse basic set of $H$.

Lemma $1 .{ }^{4}$ If $H$ is descendingly finite then every vertex of $H$ belongs to some inverse basic set of $H$.

Proof. Let $x_{1}$ be any vertex of $H$. Each descending sequence $x_{1}, x_{2}, x_{3}, \ldots$ of $H$ beginning with $x_{1}$ has a last element $x_{\lambda}$. Then

$$
x_{1} \in C^{-1}\left(x_{2}, H\right), x_{2} \in C^{-1}\left(x_{3}, H\right), \cdots, x_{\lambda-1} \in C^{-1}\left(x_{\lambda}, H\right)
$$

but

$$
x_{2} \notin C^{-1}\left(x_{1}, H\right), x_{3} \notin C^{-1}\left(x_{2}, H\right), \cdots, x_{\lambda} \notin C^{-1}\left(x_{\lambda-1}, H\right) .
$$

Hence

$$
C^{-1}\left(x_{1}, H\right) \subset C^{-1}\left(x_{2}, H\right) \subset \ldots \subset C^{-1}\left(x_{\lambda}, H\right)
$$

and

$$
C^{-1}\left(x_{\lambda}, H\right)=\bigcup_{i=1}^{\lambda} C^{-1}\left(x_{i}, H\right)
$$

is an inverse basic set containing $x_{1}$ of which $x_{\lambda}$ is a sink.

LEMmA 2. If $H$ is descendingly finite, no proper subset $B$ of an inverse basic set $A$ is an inverse basic set.

Proof. Suppose contrarywise that $B$ were an inverse basic set and a proper subset of $A$. Let $b$ be a sink of $B$ and $a$ a sink of $A$. Then $B=C^{-1}(b, H)$ and $A=C^{-1}(a, H)$. Since $B$ is a proper subset of $A, b \neq a$ and $b \in C^{-1}(a, H)$. Since the $\operatorname{sink} b$ can have no descendant relative to $H$, we have $a \in C^{-1}(b, H)$, otherwise $a$ would be a descendant of $b$. Then $C^{-1}(a, H) \subset C^{-1}(b, H)$, or $A \subset B$. Therefore $A=B$ contrary to hypothesis.

By an inverse basis of $H$ is meant a set $S$ of vertices of $H$ such that (a) $x \in S, y \in S, x \neq y$, implies that $x$ is not chain-dominated by $y$ relative to $H$,

4 Lemmas 1-5 are duals, in an obvious sense, of Lemmas 1-5 of [4] which are in turn generalizations of theorems of König [1, pp. 88-90], for finite graphs. Lemma 2 of [4, p. 581] should be corrected by adding to its statement "if $B$ has a source", and deleting from the proof all mention of Case (c); this change does not affect the rest of [4]. 
and (b) $y \in H \cap D-S$ implies that there exists a vertex $x$ of $S$ such that $x$ is chain-dominated by $y$ relative to $H$ (that is, $y \in C^{-1}(x, H)$ ).

LEMma 3. Every descendingly finite subgraph $H$ has an inverse basis.

Proof. Let the distinct inverse basic sets of $H$ be $B_{1}, B_{2}, \cdots$, where $B_{i} \neq B_{j}$ for $i \neq j$. (The range of $i$ and $j$ is any lower segment of ordinal numbers, finite or not.) By Lemma 1 , every vertex of $H$ belongs to at least one $B_{i}$. Let $b_{i}$ be a sink of $B_{i}$. Then no $b_{i}$ chain-dominates $b_{j}, i \neq j$. For, if so, $b_{i} \in C^{-1}\left(b_{j}, H\right)$. Then $b_{i}$ has $b_{j}$ as a descendant unless $b_{j} \in C^{-1}\left(b_{i}, H\right)$; that is, unless $b_{i}$ and $b_{j}$ are cyclically related relative to $H$. In this case,

$$
C^{-1}\left(b_{i}, H\right) \subset C^{-1}\left(b_{j}, H\right) \text { and } C^{-1}\left(b_{j}, H\right) \subset C^{-1}\left(b_{i}, H\right)
$$

that is, $B_{i}=B_{j}$, a contradiction. Let $S$ be the set of $b_{i}$ 's just chosen, consisting of one sink from each inverse basic set $B_{i}$. It has just been shown that Condition (a) of the definition of inverse basis is satisfied by $S$. That Condition (b) is satisfied follows immediately from Lemma 1.

Lemma 4. If $H$ has an inverse basis $S$ and $b_{i} \in S$, then $C^{-1}\left(b_{i}, H\right)$ is an inverse basic set of which $b_{i}$ is a sink.

Proof. If not, $b_{i}$ has a descendant $p$ in $H$. That is,

$$
b_{i} \in C^{-1}(p, H) \text { but } p \notin C^{-1}\left(b_{i}, H\right) .
$$

Since $p \in H \cap \mathscr{D}$, there exists a vertex $b_{j}$ of $S$ such that $p \in C^{-1}\left(b_{j}, H\right)$. Now, $b_{j} \neq b_{i}$ since $p \notin C^{-1}\left(b_{i}, H\right)$. Hence $b_{i}$ chain-dominates $p$ which chain-dominates $b_{j}$, so that $b_{i}$ chain-dominates $b_{j}$ since chain-domination is transitive. This contradicts the fact that $b_{i}$ and $b_{j}$ both belong to the inverse basis $S$.

Lemma 5. Every inverse basis $S$ of a descendingly finite subgraph $H$ consists of one sink from each inverse basic set of $H$.

Proof. By Lemma 4, each vertex of $S$ is a sink of some inverse basic set. Two distinct vertices of $S$ cannot both be sinks of the same inverse basic set since, if so, they would be chain-dominated by each other. There remains only to show that every inverse basic set has a sink in the given basis $S$. Suppose $B$ were an inverse basic set none of the sinks of which were in $S$. Let $b$ be a sink of $B$. Since $b$ is not in $S$, there exists a vertex $b^{\circ}$ of $S$ such that $b$ chaindominates $b^{\prime}$. Hence $C^{-1}(b, H) \subset C^{-1}\left(b^{\prime} ; H\right)$. But $b$ has no descendant relative 
to $H$ since $b$ is a sink. Therefore $b^{\prime}$ and $b$ must be cyclically related relative to $H$ since, if not, $b^{\prime}$ would be a descendant of $b$. Therefore $C^{-1}\left(b^{\prime}, H\right) c$ $C^{-1}(b, H)$, so that $C^{-1}(b, H)=C^{-1}(b, H)=B$. Then $b^{\prime}$ is a sink of $B$ which does lie in $S$.

5. Progressively finite graphs. A graph $H$ is termed completely descendingly finite if and only if all its closed subgraphs are descendingly finite. A sequence $\left\{x_{n}\right\}$ of vertices of $H$ is termed a progression of $H$ if and only if $x_{n} \succ x_{n+1}$, and $\mathrm{Cl}\left(x_{n} x_{n+1}\right) \subset H$ for all $n$ (except the last if the sequence is finite). $H$ is termed progressively finite if and only if all the progressions of 11 are finite.

Lemma 6. A necessary and sufficient condition that $H$ be completely descendingly finite is that $H$ be progressively finite.

Proof. If $H$ is progressively finite then it is descendingly finite. If $H$ is progressively finite then every closed subgraph of $H$ is progressively finite. Hence if $H$ is progressively finite then it is completely descendingly finite.

If $H$ is completely descendingly finite, there can exist no infinite progression $x_{1} \succ x_{2} \succ \cdots \succ x_{n} \succ \cdots$. For, if so, the subgraph consisting of the vertices $x_{i}$ and the oriented $\operatorname{arcs} x_{i} x_{i+1}(i=1,2,3, \ldots)$ would constitute a closed subgraph which would not be descendingly finite. This completes the proof.

For example, the graph $G$ of Figure 1 is descendingly finite but not completely descendingly finite since $G-\operatorname{St}(y)$ is an infinite progression.

We suppose henceforth that $\mathrm{Cl}\left(G-G_{0}\right)$ is progressively finite, where $G_{0}$ is a conjunct closed subgraph of $G$ having the solution $V_{0}$. Let ${ }^{5}$

$$
W_{00}=D\left(V_{0}, G_{0}\right) \text { and } \mathbb{W}_{0}=D\left(V_{0}, G\right) \cup D^{-1}\left(V_{0}, G-G_{0}\right) \text {. }
$$

Let

$$
G_{-1}=G-\mathrm{St}_{\mathrm{t}}\left(V_{0} \cup \mathbb{W}_{0}\right) \text {. }
$$

Let $V_{-1}$ be an inverse basis of $G_{-1}$ which exists by Lemma 3 . For each finite ordinal number $k \geq 1$, let

$$
W_{-k}=D\left(V_{-k}, G_{-k}\right) \cup D^{-1}\left(V_{-k}, G_{-k}\right),
$$

${ }^{5}$ This is a slight modification of the notation of $[4]$. 


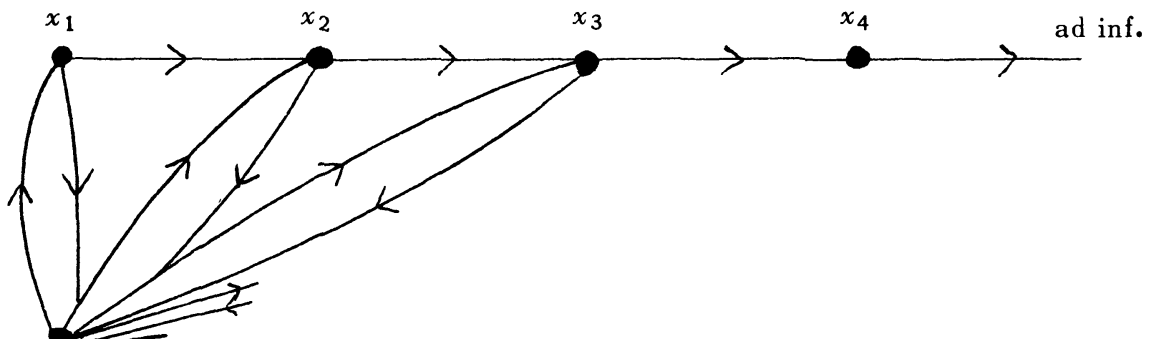

Figure 1 
and

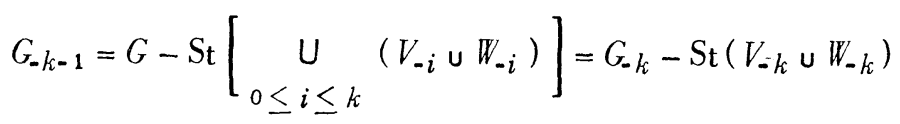

and let $V_{-k-1}$ be an inverse basis of $G_{-k-1}$.

LEMMA 7. $G_{-k-1}$ is a conjunct subgraph of $G$ for all $k \geq 0$.

Proof. Any arc of $G$ not in $G_{-k-1}$ lies in

$$
\operatorname{St}\left[\underset{i \leq k}{\cup}\left(V_{-i} \cup \mathbb{W}_{-i}\right)\right]
$$

and hence has at least one endpoint in this star. Thus if $x$ and $y$ are vertices of $G_{-k-1}$ and $x>y$ relative to $G$ then $x>y$ relative to $G_{-k-1}$ since arc $x y$ cannot lie in the star while both endpoints are in $G_{-k-1}$.

Lemma 8. For all $k \geq 0$,

$$
\bigcup_{0 \leq i \leq k+1}^{U} V_{-i}
$$

is internally satisfactory.

Proof. We prove the lemma by mathematical induction.

For $k=0$, we must prove that

$$
\left(V_{0} \cup V_{-1}\right) \cap D\left(V_{0} \cup V_{-1}, G\right)=0 \text {. }
$$

(1) $x, y \in V_{0}$ implies $x \nsucc y$ relative to $G$; for $x \nsucc y$ relative to $G_{0}$ since $V_{0}$ is a solution of $G_{0}$ and $G_{0}$ is a conjunct subgraph of $G$.

(2) $x \in V_{0}, y \in V_{-1}$ implies $x \ngtr y$ relative to $G$; for $D\left(V_{0}, G-G_{0}\right) \cap G_{-1}=0$ by definition of $G_{-1}$ while $V_{-1} \subset G_{-1}$.

(3) $x \in V_{-1}, y \in V_{0}$ implies $x \ngtr y$ relative to $G$; for $D^{-1}\left(V_{0}, G-G_{0}\right) \cap G_{-1}=0$ by definition of $G_{-1}$ while $V_{-1} \subset G_{-1}$.

(4) $x, y \in V_{-1}$ implies $x \ngtr y$ relative to $G$; for $V_{-1}$ is an inverse basis of $G_{-1}$ which implies $x \rtimes y$ relative to $G_{-1}$ while $G_{-1}$ is a conjunct subgraph of $G$ by Lemma 7 .

Assuming that $U_{i \leq k} V_{-i}$ is internally satisfactory, we complete the proof by 
showing:

(a)

$$
V_{-k-1} \cap D\left(\bigcup_{i \leq k}^{\cup} V_{-i}, G\right)=0 ;
$$

$$
V_{-k-1} \cap D\left(V_{-k-1}, G\right)=0 \text {; }
$$

(c)

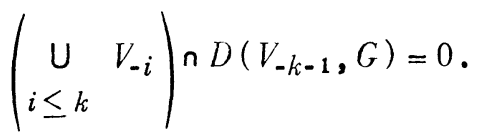

If

$$
x \in \underset{i \leq k}{\bigcup} V_{-i}, y \in V_{-k-1},
$$

then $x \ngtr y$; for if $x \succ y$ then

$$
y \in \underset{j \leq k}{U} \mathbb{W}_{-j}
$$

and $y \notin G_{-k-1}$, while $V_{-k-1} \subset G_{-k-1}$. This proves (a). Since $G_{-k-1}$ is a conjunct subgraph of $G, x \succ y$ relative to $G$, where $x, y \in V_{-k-1}$, would imply $x>y$ relative to $G_{-k-1}$, contrary to the definition of inverse basis. This proves (b). If

$$
x \in V_{-k-1}, y \in \underset{i \leq k}{\bigcup} V_{-i},
$$

then $x \ngtr y$; for if $x \succ y$ then

$$
x \in \underset{i \leq k}{\cup} D^{-1}\left(V_{-i}, G_{-i}\right) \subset \underset{i \leq k}{\bigcup} \mathbb{W}_{-i}
$$

so that $x \notin G_{-k-1}$, a contradiction. This completes the proof.

It may happen that $G_{-n}=0$ for no finite ordinal $n$, in which case we may let

$$
G_{-\omega}=G-\mathrm{St}\left[\underset{i<\omega}{U}\left(V_{-i} \cup \mathbb{W}_{-i}\right)\right],
$$

$V_{-\omega}=$ any inverse basis of $G_{-\omega}$, and

$$
W_{-\omega}=D\left(V_{-\omega}, G_{-\omega}\right) \cup D^{-1}\left(V_{-\omega}, G_{-\omega}\right),
$$

and so on. Transfinite induction shows that if $\beta$ is an ordinal number for which 
$V_{-\alpha}$ is nonempty for all $\alpha<\beta$ then

$$
\underset{\alpha<\beta}{U} V_{-\alpha}
$$

is internally satisfactory. Let the cardinal number of the set $\mathscr{D}$ be $\boldsymbol{x}_{\mu}$. Let $\lambda$ be the next largest ordinal after those of $\supset\left(\boldsymbol{x}_{\mu}\right)$ where $\supset\left(\boldsymbol{x}_{\mu}\right)$ is the set of all ordinal numbers of well-ordered sets having cardinal number $\boldsymbol{x}_{\mu}$. Then no matter how we well-order the elements of $\mathscr{\partial}$, its ordinal number is $<\lambda$. Wellorder them as follows:

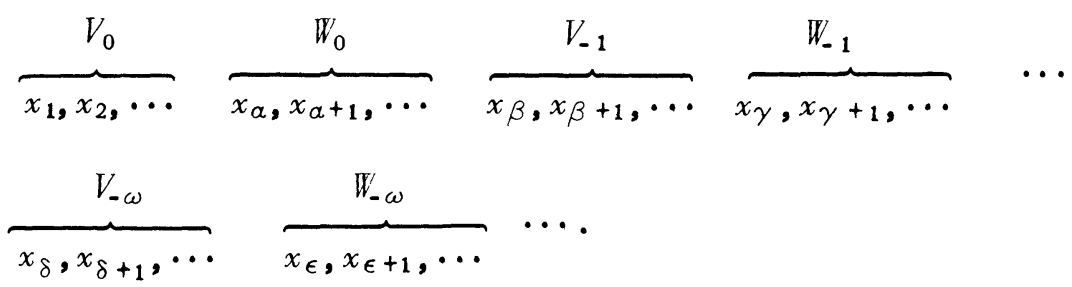

Then every vertex of $\mathcal{D}$ is in some $V_{-\zeta}$ or some $W_{-\zeta}$ with $\zeta<\lambda$. Let $\kappa$ be the lowest ordinal for which $G_{-}=0$. Then every vertex of $G$ is ultimately used up in some $V_{-\zeta}$ or $W_{-}, \zeta<\kappa$. We have then the following theorems in which we let

$$
V=\underset{0 \leq \alpha<\kappa}{U} V_{-\alpha}
$$

THEOREM 3. If $V_{0}$ is a solution of the subsystem $\left(\mathscr{D}_{0}, \succ\right)$ of the system $(\supseteq, \succ)$, and if the graph $\mathrm{Cl}\left(G-G_{0}\right)$ is progressively finite, and every vertex of $G-G_{0}$ is in the predecessor-set $P\left(\Phi_{0}, G-G_{0}\right)$, then $V$ is a maximally internally satisfactory set.

THEOREM 4. If, in addition to the hypotheses of Theorem 3, there exist inverse bases $V_{-a}$ for each $\alpha$ with $1 \leq \alpha<\kappa$ such that

$$
D^{-1}\left(V_{0}, G-G_{0}\right) \subset D(V, G) \text { and } D^{-1}\left(V_{-\alpha}, G_{-a}\right) \subset D(V, G) \text {, }
$$

then $V$ is a solution of $G$ and an extension of $V_{0}$.

THEOREM 5. If, in addition to the hypotheses of Theorem $3, \succ$ is symmetric, then $V$ is a solution of $G$ and an extension of $V_{0}$.

The proofs of Theorems 4 and 5 are immediate. ${ }^{6}$

6 As to Theorem 5 , the fact that if $>$ is symmetric then every maximally internally satisfactory set is a solution is established in [ 2 ]. 
THEOREM 6. If the hypotheses of Theorem 2 are satisfied, then so are the hypotheses of Theorem 4.

$$
\begin{aligned}
& \text { Proof. Let } \\
& W_{0}=D\left(V_{0}, G\right) \cup D^{-1}\left(V_{0}, G-G_{0}\right), \\
& V_{-1}=D^{-2}\left(V_{0}, G-G_{0}\right) \cup D^{-1}\left(W_{00}, G-G_{0}\right), \\
& W_{-1}=D^{-3}\left(V_{0}, G-G_{0}\right) \cup D^{-2}\left(W_{00}, G-G_{0}\right)=D^{-1}\left(V_{-1}, G-G_{0}\right) \cup D\left(V_{-1}, G-G_{0}\right), \\
& V_{-2}=D^{-4}\left(V_{0}, G-G_{0}\right) \cup D^{-3}\left(\mathbb{W}_{00}, G-G_{0}\right),
\end{aligned}
$$

and so on. Then

$$
V=\underset{0 \leq a}{\cup} V_{-a}=V_{0} \cup \cup D^{-2 n}\left(V_{0}, G-G_{0}\right) \cup \cup D^{-2 n+1}\left(\mathbb{W}_{00}, G-G_{0}\right)
$$

and

$$
\mathbb{W}=\underset{0 \leq a}{\cup} \mathbb{W}_{-a}=\mathbb{W}_{00} \cup \cup D^{-2 n+1}\left(V_{0}, G-G_{0}\right) \cup \cup D^{-2 n}\left(W_{00}, G-G_{0}\right),
$$

so that $V$ is a solution.

There remains to show that $V_{-a}$ is an inverse basis of $G_{-a}$. Clearly, neither of two distinct vertices $x, y \in V_{-a}$ chain-dominates the other by virtue of the parity restrictions (2), ( 3 ) of Theorem 2 . We must show now that every vertex $y$ of $G_{-\alpha}$ chain-dominates some $x$ of $V_{-a}$. This is obvious since by (4) every $y$ belongs to $P\left(I_{0}, G-G_{0}\right)$, that is, to some $D^{-n}\left(V_{0}, G-G_{0}\right)$ or to some $D^{-n}\left(W_{00}\right.$, $\left.G-G_{0}\right)$, that is, to some $V_{-\alpha}$ or $W_{-\alpha}$. By $(1)$ it is clear that every $D^{-1}\left(V_{-\alpha}, G_{-\alpha}\right) C$ $D(V, G)$. This completes the proof.

The example of Figure 2 shows that Theorem 4 is less restrictive than Theorem 2. For

$$
w_{0}^{2} \in D^{-1}\left(W_{00}, G-G_{0}\right) \cap D^{-2}\left(V_{0}, G-G_{0}\right) \cap D^{-1}\left(V_{0}, G-G_{0}\right)
$$

but an extension exists and the hypotheses of Theorem 4 are satisfied.

6. Some extension theorems. If $H$ is a subgraph of $G$, let

$$
K(x, H)=D(x, H) \cup D^{-1}(x, H), \quad x \in \supseteq ;
$$




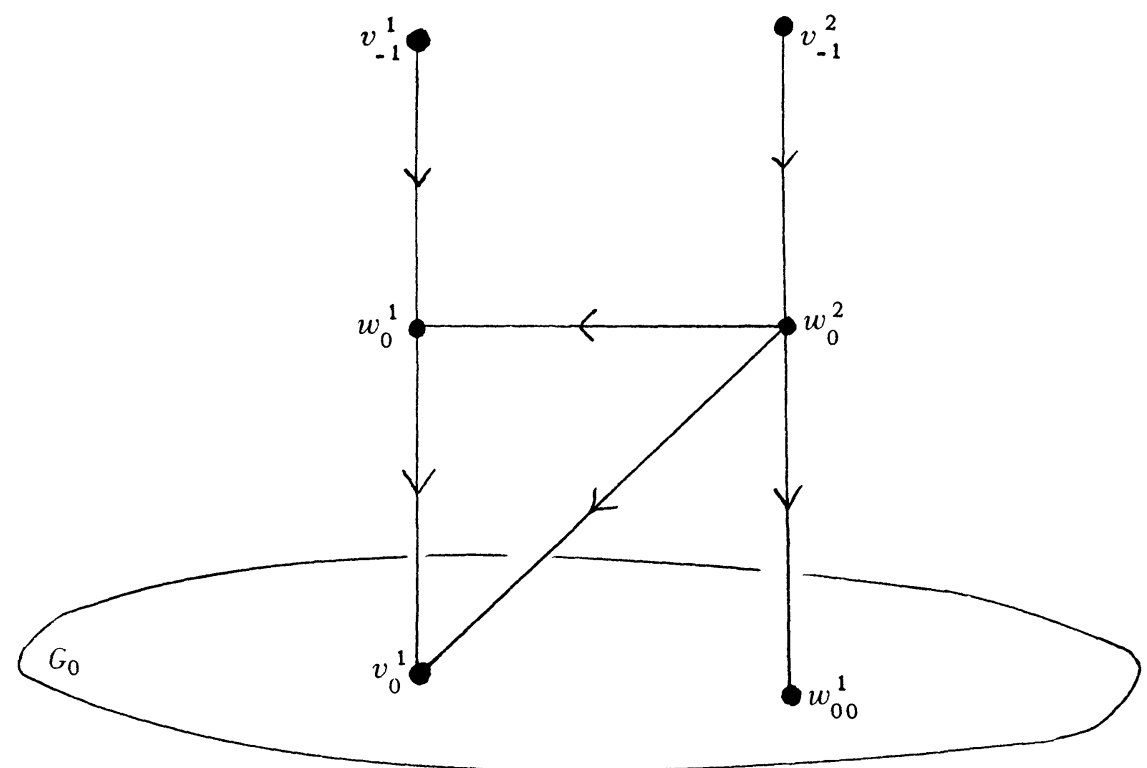

Figure 2 
let

$$
K(X, H)=\bigcup_{x \in X} K(x, H),
$$

let

$$
K^{n}(X, H)=K\left(K^{n-1}(X, H), H\right) \quad \text { for } n>1 \text {. }
$$

That is, $K^{n}(X, H)$ denotes the set of vertices of $H$ connected to vertices of $X$ by unoriented one-dimensional chains of length $n$.

Lemma 9. If $\supseteqq-\mathscr{D}_{0} \subset P\left(V_{0}, G-G_{0}\right)$, then every inverse basic set $B$ of $G_{-i-1}$ has a sink in $K^{2}\left(V_{-i}, G_{-i-1}\right), i \geq 0$.

Proof. Suppose $i=0$. Each sink $y$ of $B$ chain-dominates some vertex of $V_{0}$ since $\supseteq-\oslash_{0} \subset P\left(V_{0}, G-G_{0}\right)$. Consider the chains of minimum length $m$ by which $y$ chain-dominates vertices of $V_{0}$. Then $m \geq 2$ since $K\left(V_{0}, G-G_{0}\right) n$ $G_{-1}=0$. Suppose the lemma were false, so that $m>2$, and let $y_{0}$ be a sink of $B$ for which this minimum length is attained. Then there exist distinct vertices $x_{1}, x_{2}, \cdots, x_{m-1}$ of $G-G_{0}$ such that

$$
y_{0} \succ x_{m-1} \succ x_{m-2} \succ \cdots \succ x_{1} \succ v_{0}^{j}
$$

for some $v_{0}^{j} \in V_{0}$. Then either

(1) $x_{m-1} \notin G_{-1}$,

or (2) $x_{m-1} \in G_{-1}$ and is a descendant of $y_{0}$,

or (3) $x_{m-1} \in G_{-1}$ and is cyclically related to $y_{0}$ relative to $G_{-1}$.

In Case (1), $x_{m-1} \in V_{0} \cup W_{0}$ so that

$$
x_{m-1} \in K^{1}\left(V_{0}, G_{-1}\right) \text { and } y_{0} \in K^{2}\left(V_{0}, G_{-1}\right)
$$

contrary to the supposition that the lemma is false. In Case (2) $y_{0}$ is not a sink of $B$ since a sink can have no descendant. In Case (3), $m$ is not the minimum length since $x_{m-1}$ would be a sink of $B$ which chain-dominates $v_{0}^{j}$ by means of a chain of length $m-1$.

Now suppose $i>0$. Let $B$ be an inverse basic set of $G_{-i-1}$. Each sink $y$ of $B$ chain-dominates some vertex of $V_{-i}$ since $V_{-i}$ is an inverse basis of $G_{-i} \supset G_{-i-1}$. 
Consider the chains of minimum length $m$ by which $y$ chain-dominates vertices of $V_{-i}$. Then $m \geq 2$ since $K\left(V_{-i}, G_{-i}\right) \cap G_{-i-1}=0$. Suppose the lemma were false, so that $m>2$, and let $y_{0}$ be a sink of $B$ for which this minimum length is attained. Then there exist distinct vertices $x_{1}, x_{2}, \cdots, x_{m-1}$ of $G_{-i}$ such that

$$
y_{0} \succ x_{m-1} \succ x_{m-2} \succ \cdots \succ x_{1} \succ v_{-i}^{j} \text { for some } v_{-i}^{j} \in V_{-i}
$$

Then either

(1) $x_{m-1} \notin G_{-i-1}$,

or (2) $x_{m-1} \in G_{-i-1}$ and is a descendant of $y_{0}$,

or (3) $x_{m-1} \in G_{-i-1}$ and is cyclically related to $y_{0}$ relative to $G_{-i-1}$. In Case ( 1 ),

$$
x_{m-1} \in \operatorname{St}\left(V_{-i} \cup \mathbb{W}_{-i}^{\prime}\right)
$$

and hence $x_{m-1} \in V_{-i} \cup \mathbb{W}_{-i}$ and hence $x_{m-1} \in K^{1}\left(V_{-i}, G_{-i}\right)$ so that $y_{0} \in K^{2}\left(V_{-i}\right.$, $\left.G_{-i-1}\right)$ contrary to our supposition that the lemma is false. In Case (2), $y_{0}$ is not a sink of $B$ since a sink has no descendant. In Case (3), $m$ is not minimal since $x_{m-1}$ would be a sink of $B$ which chain-dominates $v_{-i}^{j}$ by means of a chain of length $m-1$. This completes the proof.

The example of Figure 3 shows that we must take $K^{n}$ in the unoriented sense; for here $v_{-1}^{1} \in P\left(V_{0}, G-G_{0}\right)$, in fact $v_{-1}^{1} \in D^{-4}\left(V_{0}, G-G_{0}\right)$ but $v_{-1}^{1} \notin$ $D^{-2}\left(V_{0}, G-G_{0}\right)$ although $v_{-1}^{1} \in K^{2}\left(V_{0}, G-G_{0}\right)$.

A subgraph $H$ of $G$ is termed progressively bounded at the vertex $y$ if all progressions of $H$ beginning with $y$ have lengths forming a bounded set of natural numbers. $H$ is termed progressively bounded if it is progressively bounded at each of its vertices.

Lemma 10. If $\supseteq-D_{0} \subset P\left(V_{0}, G-G_{0}\right)$ and if $\mathrm{Cl}\left(G-G_{0}\right)$ is progressively bounded then every vertex $y$ of $\mathscr{P}-\mathscr{S}_{0}$ is an element of $V_{-i}$ or $W_{-i}$ for some finite ordinal $i$.

Proof. Every vertex $y$ of $\mathscr{D}-D_{0}$ is an element of $C^{-1}\left(v_{0}^{j}, \mathrm{Cl}\left(G-G_{0}\right)\right)$ for some $v_{0}^{j} \in V_{0}$ by hypothesis. Consider all progressions of $\mathrm{Cl}\left(G-G_{0}\right)$ beginning with $y$ and ending with elements of $V_{0}$. Their lengths have a least upper bound $M(y)$ by hypothesis. By Lemma 9, we may select inverse bases

$$
V_{-i-1} \subset K^{2}\left(V_{-i}, G-G_{-i-1}\right),
$$




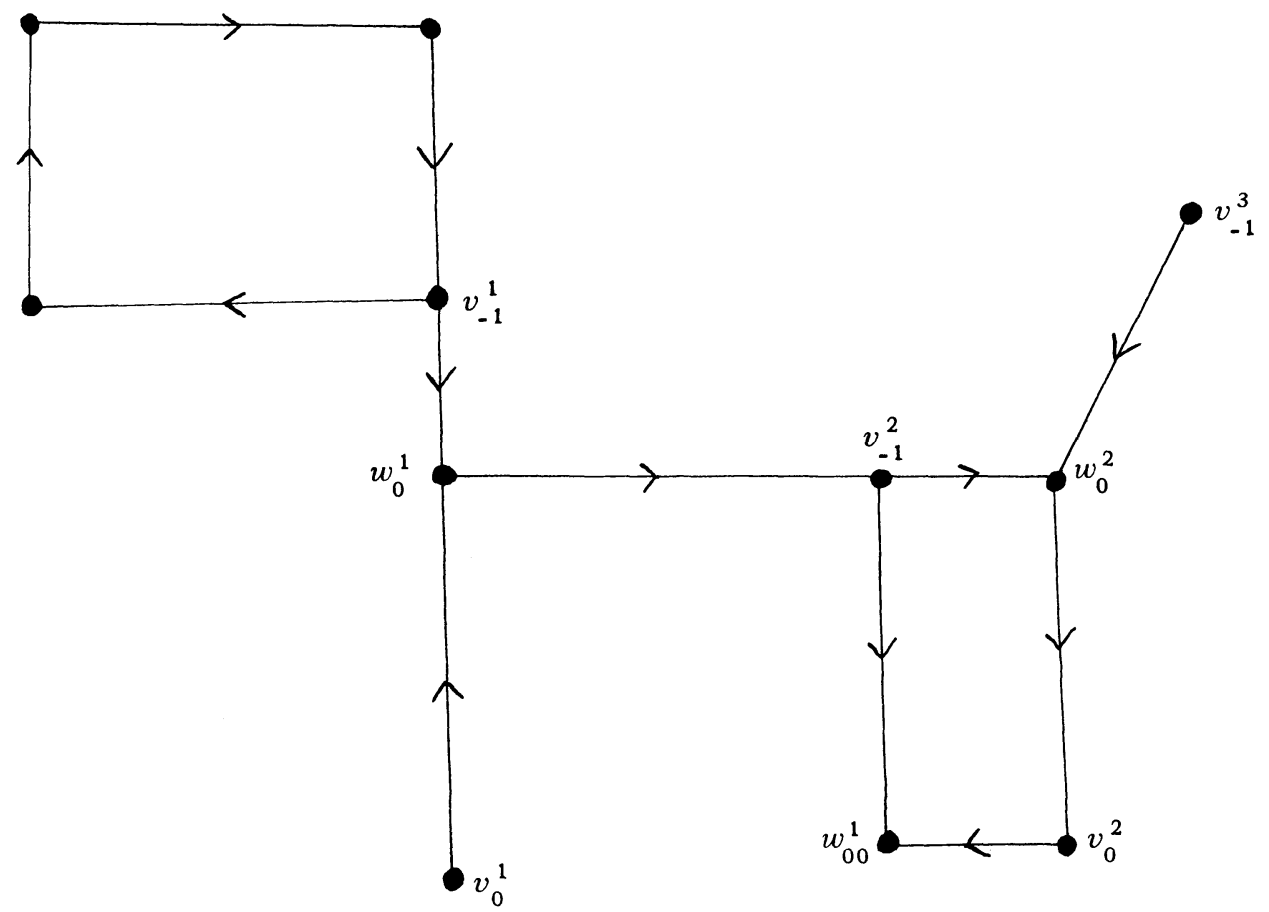

Figure 3 
Since $V_{-1}$ is an inverse basis of $G_{-1}$, there exist progressions starting with $y$ and ending with elements of $V_{-1}$ unless $y$ is in $V_{-k}$ or $W_{-k}$ with $k \leq 1$. All such progressions have lengths $\leq M(y)-2$. For if there existed a progression from $y$ to some $v_{-1}^{h} \in V_{-1}$ of length $>M(y)-2$, there would be a progression from $y$ to some element of $V_{0}$ of length $>M(y)$ since there exists some progression from $v_{-1}^{h}$ to some element of $V_{0}$ and its length must be $\geq 2$ because $V_{-1} \subset G_{-1}$. Similarly the lengths of all progressions from $y$ to elements of $V_{-i}$ must be $\leq M(y)-2 i$. But this can be $\geq 0$ for only a finite number of values of $i$. Hence there exists a value of $i$ for which $y$ chain-dominates some element of $V_{-i}$ by means of a progression of length 0 or 1 ; that is, $y$ is in either $V_{-i}$ or $W_{-i}$.

By a relative cycle ( of $\mathrm{Cl}\left(G-G_{0}\right) \bmod V_{0}$ with modulo 2 coefficients) shall be meant an unoriented one-dimensional chain lying in $\mathrm{Cl}\left(G-G_{0}\right)$ except for its set of boundary vertices (possibly empty; that is, absolute cycles are included among the relative cycles) which lies in $V_{0}$.

THEOREм 7. Suppose that $V_{0}$ is a solution of $G_{0}$ such that .

(1) $\mathrm{Cl}\left(G-G_{0}\right)$ is progressively bounded,

(2) each vertex of every $K^{2 n-1}\left(V_{0}, G-G_{0}\right)$ is dominated by some element of $\supseteq \mathscr{D}_{0}$;

(3) $\mathrm{Cl}\left(G-G_{0}\right)$ contains no relative cycle of odd length;

(4) $\supseteq D_{0} \subset P\left(V_{0}, G-G_{0}\right)$.

Then there exists a solution $V$ of $G$ which is an extension of $V_{0}$.

Proof. Choose $V_{-i}$ as in Lemma 9. To show that $V=\mathrm{U}_{0} \leq_{i} V_{-i}$ is a solution of $G$ we have, by Theorem 4, only to show that

$$
D^{-1}\left(V_{0}, G-G_{0}\right) \subset D(V, G) \text { and } D^{-1}\left(V_{-i}, G_{-i}\right) \subset D(V, G) \text { for } \quad i \geq 1
$$

Let

$$
w \in D^{-1}\left(V_{0}, G-G_{0}\right) \cup D^{-1}\left(V_{-i}, G_{-i}\right) .
$$

Then

$$
w \in K^{2 n-1}\left(v_{0}^{j}, G-G_{0}\right)
$$


for some $j$ and $n$ by virtue of the way in which the $V_{-i}$ were chosen. By (2), $w$ is dominated by some vertex $x$ of $\mathscr{S}-\mathscr{D}_{0}$. If $x \in V$, there is no more to prove. If $x \in \mathfrak{D}-V$, then

$$
x \in K^{2 m-1}\left(v_{0}^{k}, G-G_{0}\right)
$$

for some $k$ and $m$. Hence there exists a relative cycle of odd length, contrary to (3). This completes the proof.

THEOREM 8. Let $V$ be any maximally internally satisfactory set containing $V_{0}$ such that:

(1) every $v \in V$ belongs to $K^{2 n}\left(V_{0}, G-G_{0}\right)$ for some $n \geq 0$;

(2) each element of $K^{2 m-1}\left(V_{0}, G-G_{0}\right)$, for every $m>0$, is dominated by some element of $\supseteqq-D_{0}$;

(3) $\mathrm{Cl}\left(G-G_{0}\right)$ contains no relative cycle of odd length.

Then $V$ is a solution of $G$.

Proof. Let

$$
y \in\left(\mathscr{P}-\mathscr{\vartheta}_{0}\right) \cap(\mathscr{P}-V) .
$$

We shall show that there exists an $x \in V$ such that $x \succ y$. Since $V$ is maximally internally satisfactory, $V \cup(y)$ is not internally satisfactory. Therefore either (a) some $v \succ y$, or (b) some $v \prec y$. In Case (a), there is no more to prove. In Case (b),

$$
y \in K^{2 n-1}\left(V_{0}, G-G_{0}\right) \quad \text { for some } n \geq 0 \text {. }
$$

By (2), there exists an $x \in \mathscr{D}-\mathscr{D}_{0}$ such that $x>y$. If $x \in V$, there is no more to prove. If not, that is if $x \in\left(\mathscr{D}-\mathscr{D}_{0}\right) \cap(\mathscr{D}-V)$, then $V \mathrm{u}(x)$ is not internally satisfactory. Therefore there exists a $v \in V$ such that either $x \succ v$ or $x \prec v$. In either case,

$$
x \in K^{2 m-1}\left(V_{0}, G-G_{0}\right)
$$

for some natural number $m$. But this together with

$$
y \in K^{2 n-1}\left(V_{0}, G-G_{0}\right)
$$


and $x>y$ imply that there exists a relative cycle of odd length contrary to (3). This completes the proof.

COROLLARY. The hypotheses of Theorem 8 imply that

$$
V=\cup K^{2 n}\left(V_{0}, G-G_{0}\right) \text { and } W=\mathfrak{I}-V=\cup K^{2 m-1}\left(V_{0}, G-G_{0}\right) \text {. }
$$

Proof. We have

$$
V \subset \cup K^{2 n}\left(V_{0}, G-G_{0}\right)=E,
$$

and

$$
W=\mathfrak{D}-V \subset \cup K^{2 m-1}\left(V_{0}, G-G_{0}\right)=\Omega \text {. }
$$

Furthermore

$$
K^{2 n}\left(V_{0}, G-G_{0}\right) \cap K^{2 m-1}\left(V_{0}, G-G_{0}\right)=0,
$$

for, if not, there would exist a relative cycle of odd length. Thus we have

$$
E \cap \Omega=0, E \cup \Omega=\mathscr{P}, V \subset E, \mathbb{W} \subset \Omega, V \cup \mathbb{W}=\mathscr{S} \text {, and } V \cap \mathbb{W}=0 \text {. }
$$

This implies $E=V, \Omega=\mathbb{W}$ as follows. Let $e \in E$. Then $e \in \mathscr{D}$ which implies that either $e \in V$ or $e \in W$. But $e \in \mathbb{W}$ would imply that $e \in \Omega$ contrary to $E \cap \Omega=0$. Therefore $e \in V$. Hence $E \subset V$ and therefore $E=V$. Similarly $\Omega \subset W$ and hence $\Omega=W$. This completes the proof.

Thus Theorem 8 resembles Theorem 2, except that now the parity restrictions are on the unoriented chains rather than on the oriented ones, and we do not restrict the sets $K^{n}\left(W_{00}, G-G_{0}\right)$.

The examples of Figures $4-6$ are covered by Theorem 8 but not by Theorem 2. In Figure 4,

$$
w_{0}^{1} \in L\left(V_{0}, G\right) \cap D^{-1}\left(\mathbb{W}_{00}, G-G_{0}\right) \neq 0
$$

violating hypothesis $2 \mathrm{~b}$ of Theorem 2 , but the extension exists under Theorem 8. In Figure 5,

$$
v_{-1}^{1} \in D^{-2}\left(W_{00}, G-G_{0}\right) \cap D^{-1}\left(W_{00}, G-G_{0}\right) \neq 0
$$

violating the second part of the hypothesis $3 \mathrm{~b}$ of Theorem 2, but the extension 


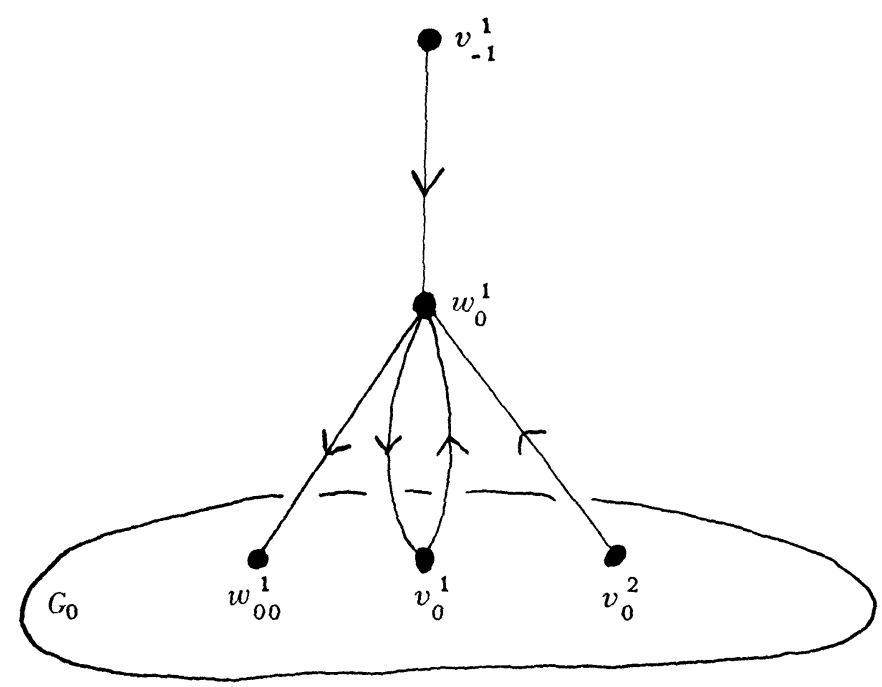

Figure 4 


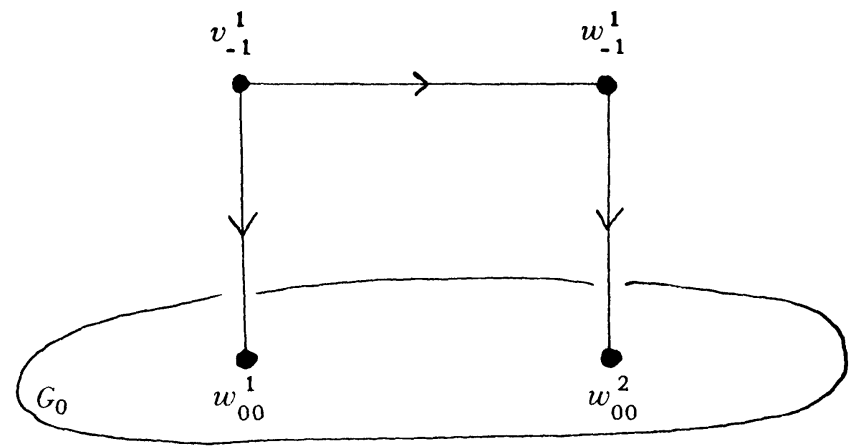

Figure 5 


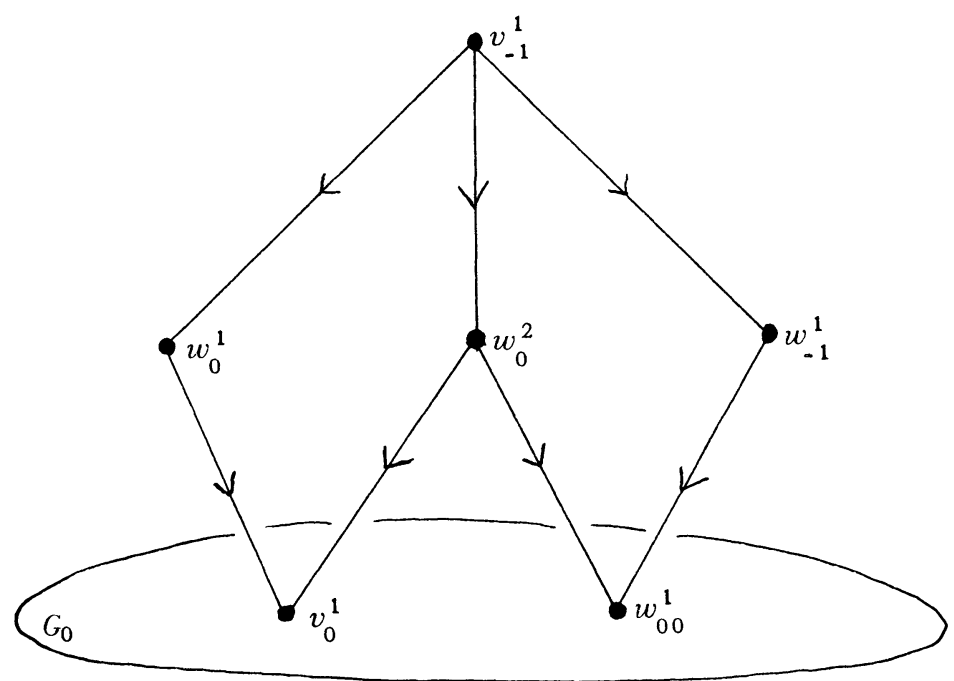

Figure 6 
exists under Theorem 3 . Note also that an odd relative cycle exists mod $G_{0}$ but not $\bmod V_{0}$. In Figure 6,

$$
v_{-1}^{1} \in D^{-2}\left(V_{0}, G-G_{0}\right) \cap D^{-2}\left(W_{00}, G-G_{0}\right) \neq 0
$$

and

$$
w_{0}^{2} \in D^{-1}\left(V_{0}, G-G_{0}\right) \cap D^{-1}\left(W_{00}, G-G_{0}\right) \neq 0
$$

both violating hypothesis $3 \mathrm{a}$ of Theorem 2, but the extension exists under Theorem 8.

Let $\mu^{h}\left(X, G-G_{0}\right)$ denote the set of vertices of $G-G_{0}$ connected to $X$ by an unoriented chain of minimal length $h$, where $X \subset \mathcal{S}$. Then

$$
\mu^{h}\left(X, G-G_{0}\right) \cap \mu^{k}\left(X, G-G_{0}\right)=0 \quad \text { for } h \neq k \text {. }
$$

By $\mu^{0}\left(X, G-G_{0}\right)$ is meant $X$.

THEOREM 9. Let $V_{0}$ be a solution of $G_{0}$ where $G_{0}$ is a conjunct subgraph of $G$. Let $W_{00}=\mathscr{D}_{0}-V_{0}$ and suppose that every vertex of $\mathscr{D}_{0} \mathscr{D}_{0}$ is connected to $D_{0}$ by some unoriented chain. Let

$$
\begin{aligned}
& V=\bigcup_{n=0}^{\infty} \mu^{2 n}\left(V_{0}, G-G_{0}\right) \cup \underset{m=1}{\bigcup_{m}^{\infty}} \mu^{2 m-1}\left(W_{00}, G-G_{0}\right), \\
& W=\bigcup_{n=1}^{\infty} \mu^{2 n-1}\left(V_{0}, G-G_{0}\right) \cup \underset{m=0}{\bigcup} \mu^{2 m}\left(W_{00}, G-G_{0}\right) .
\end{aligned}
$$

Suppose that:

(1) every element of $W$ is dominated by some element of $V$;

(2) $\mu^{h}\left(V_{0}, G-G_{0}\right) \cap \mu^{k}\left(W_{00}, G-G_{0}\right)=0$

if $h$ and $k$ have the same parity;

(3) no two elements of the same $\mu^{2 n-1}\left(W_{00}^{\prime}, G-G_{0}\right)$ are adjacent;

(4) no two elements of the same $\mu^{2 n}\left(V_{0}, G-G_{0}\right)$ are adjacent.

Then $V$ is a solution of $G$ which is an extension of $V_{0}$. 
Proof. Clearly $\supseteqq=V \cup W$ and $W \subset D(V, G)$. Also (2) implies $V \cap W=0$. There remains only to prove that no two elements of $V$ are adjacent.

If $x, y \in \mu^{2 n}\left(V_{0}, G-G_{0}\right)$ then $x \ngtr y$ by $(4)$.

If $x, y \in \mu^{2 m-1}\left(W_{00}, G-G_{0}\right)$ then $x \ngtr y$ by ( 3$)$.

Let

$$
x \in \mu^{2 n}\left(V_{0}, G-G_{0}\right), y \in \mu^{2 m}\left(V_{0}, G-G_{0}\right), \quad m \neq n .
$$

Suppose $m>n$. If $x$ and $y$ were adjacent then $y \in K^{2 n+1}\left(V_{0}, G-G_{0}\right)$. But $2 n+1<2 m$, contradicting the minimal property of $\mu^{2 m}\left(V_{0}, G-G_{0}\right)$. A similar proof is obtained if $m<n$.

If

$$
x \in \mu^{2 n-1}\left(\mathbb{W}_{00}, G-G_{0}\right), y \in \mu^{2 m-1}\left(\mathbb{W}_{00}, G-G_{0}\right), \quad m \neq n,
$$

then $x$ and $y$ are proved non-adjacent as in the preceding paragraph.

Let

$$
x \in \mu^{2 n}\left(V_{0}, G-G_{0}\right), y \in \mu^{2 p-1}\left(W_{00}, G-G_{0}\right)
$$

and suppose $x$ were adjacent to $y$. Then

$$
x \in K^{2 p}\left(W_{00}, G-G_{0}\right) \text { or } x \in K^{2 p-2}\left(W_{00}, G-G_{0}\right) .
$$

Since $x$ is connected to $W_{00}$, it is minimally connected to $W_{00}$. That is, either

$$
x \in \mu^{2 h}\left(W_{00}, G-G_{0}\right)
$$

or

$$
x \in \mu^{2 h-1}\left(W_{00}, G-G_{0}\right)
$$

for some $h$. In Case (a), Condition (2) would be violated. In Case (b), $h=p$ since either $h<p$ or $h>p$ would violate the minimal property of some $\mu$. But $h=p$ contradicts Condition (3). This completes the proof.

THEOREM 10. Let $V_{0}$ be a solution of a conjunct subgraph $G_{0}$ of $G$ such that every vertex of $\supseteq-\vartheta_{0}$ is connected to $V_{0}$ by some unoriented chain. Let: 
(1) no two elements of the same $\mu^{2 i}\left(V_{0}, G-G_{0}\right), i>0$, be adjacent;

(2) $x \in \mu^{2 i-1}\left(V_{0}, G-G_{0}\right)$ imply that there exists $a j \geq 0$ such that $x \prec y$ for some $y \in \mu^{2 j}\left(V_{0}, G-G_{0}\right)$.

Then

$$
V=\bigcup_{i=0}^{\infty} \mu^{2 i}\left(V_{0}, G-G_{0}\right)
$$

is a solution of $G$ which is an extension of $V_{0}$.

Proof. Every element of $\mathscr{O}-\mathscr{D}_{0}$ not in $V$ must be in

$$
W=\bigcup_{i=1}^{\infty} \mu^{2 i-1}\left(V_{0}, G-G_{0}\right)
$$

Clearly

$$
\Phi=V \cup W \text { and } V \cap \mathbb{W}=0 \text {. }
$$

Also (2) implies $W \subset D(V, G)$. There remains only to prove that $V$ is internally satisfactory.

Let

$$
x \in \mu^{2 i}\left(V_{0}, G-G_{0}\right), y \in \mu^{2 j}\left(V_{0}, G-G_{0}\right),
$$

Suppose $i<j$. If $x$ were adjacent to $y$, then $y \in K^{2 i+1}\left(V_{0}, G-G_{0}\right)$. But $2 i+1<$ $2 j$, contradicting the minimal property of $\mu^{2 j}\left(V_{0}, G-G_{0}\right)$. A similar proof holds if $i>j$.

Let $x, y \in \mu^{2 i}\left(V_{0}, G-G_{0}\right)$. If $i>0$, (1) implies that $x$ and $y$ are nonadjacent. For $i=0$, this follows from the facts that $V_{0}$ is a solution of $G_{0}$ and that $G_{0}$ is a conjunct subgraph of $G$. This completes the proof.

The conditions of Theorem 10 do not prohibit entirely the existence in $\mathrm{Cl}\left(G-G_{0}\right)$ of adjacent vertices of $W$, of odd unoriented cycles, or of transitive triples. For example, the graph in Figure 7 permits an extension by Theorem 10 and includes the three cited phenomena. Theorems 7-10 may be regarded as variants of Theorem 2 .

7. Dual and alternating procedures. Let $G_{1}$ be a conjunct subgraph of $G$. 


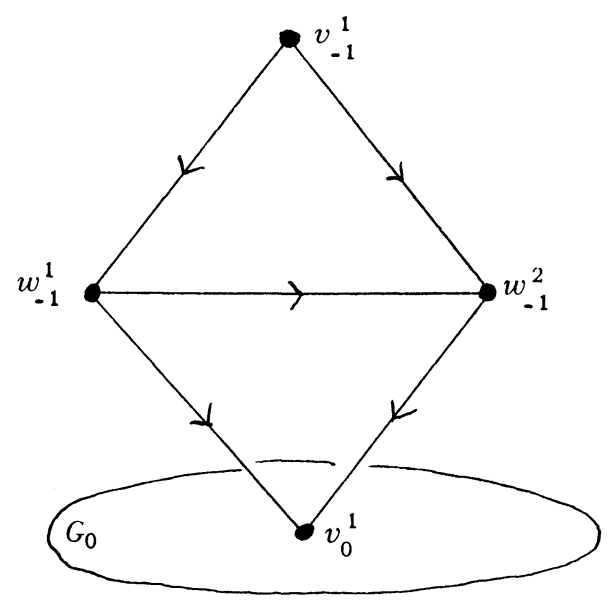

Figure 7 
If $x \in \mathcal{O}$, let $D\left(x, G-G_{1}\right)$ denote the set of all vertices $y$ of $G-G_{1}$ such that $x>y$. If $X \subset \supseteqq$, let

$$
D\left(X, G-G_{1}\right)=\underset{x \in X}{\bigcup} D\left(x, G-G_{1}\right) .
$$

For $n>1$, let

$$
D^{n}\left(X, G-G_{1}\right)=D\left(D^{n-1}\left(X, G-G_{1}\right), G-G_{1}\right) .
$$

By the successor-set of $X$ relative to $G-G_{1}$ is meant the set

$$
S\left(X, G-G_{1}\right)=\bigcup_{n=1}^{\infty} D^{n}\left(X, G-G_{1}\right) .
$$

THE OREM 11. Let $G_{1}$ be a conjunct subgraph of $G, V_{1}$ a solution of $G_{1}$, $W_{1}=D_{1}-V_{1}$ where $\mathscr{D}_{1}=\overbrace{\cap} G_{1}$. Suppose that:

(1) for every $n>0$,

$$
D\left(V_{1}, G\right) \cap D^{2 n}\left(V_{1}, G-G_{1}\right)=D\left(V_{1}, G\right) \cap D^{2 n-1}\left(W_{1}, G-G_{1}\right)=0 \text {, }
$$

and

$$
V_{1} \cap D^{2 n+1}\left(V_{1}, G-G_{1}\right)=V_{1} \cap D^{2 n}\left(W_{1}, G-G_{1}\right)=0 ;
$$

(2) if $h>0$ and $k>0$ are of the same parity, then

$$
D^{h}\left(V_{1}, G-G_{1}\right) \cap D^{k}\left(W_{1}, G-G_{1}\right)=0 ;
$$

if $h>0$ and $k>0$ are of different parities then

$$
\begin{aligned}
& D^{h}\left(V_{1}, G-G_{1}\right) \cap D^{k}\left(V_{1}, G-G_{1}\right)=D^{h}\left(W_{1}, G-G_{1}\right) \cap D^{k}\left(W_{1}, G-G_{1}\right)=0 ; \\
& \text { (3) } \oslash-\oslash_{1} \subset S\left(\oslash_{1}, G-G_{1}\right) .
\end{aligned}
$$

Then there exists a solution $V$ of $G$ which is an extension of $V_{1}$.

Proof. Let

$$
V=V_{1} \cup \underset{n=1}{\bigcup} D^{2 n}\left(V_{1}, G-G_{1}\right) \cup \underset{m=1}{\bigcup} D^{2 m-1}\left(W_{1}, G-G_{1}\right) .
$$


We must show:

$$
V \cap D(V, G)=0
$$

$$
\text { D }-V \subset D(V, G) \text {. }
$$

(a) If $x \in V_{1}, y \in V_{1}$, then $x \ngtr y$ since $V_{1}$ is internally satisfactory relative to $G$.

If $x \in V_{1}, y \in D^{2 n}\left(V_{1}, G-G_{1}\right)$ then $x . y$; for $x>y$ would imply $D\left(V_{1}, G\right)$ n $D^{2 n}\left(V_{1}, G-G_{1}\right) \neq 0$ contrary to $(1)$.

If $x \in V_{1}, y \in D^{2 m-1}\left(W_{1}, G-G_{1}\right)$ then $x \nsucc y$; for $x \succ y$ would imply $D\left(V_{1}\right.$, $G) \cap D^{2 m-1}\left(W_{1}, G-G_{1}\right) \neq 0$ contrary to (1).

If $x \in D^{2 n}\left(V_{1}, G-G_{1}\right), y \in V_{1}$, then $x \nsucc y$; for $x \succ y$ would imply $y \in$ $D^{2 n+1}\left(V_{1}, G-G_{1}\right)$ contrary to $(1)$.

If $x \in D^{2 n}\left(V_{1}, G-G_{1}\right), y \in D^{2 m}\left(V_{1}, G-G_{1}\right)$ then $x \nsucc y$; for $x \succ y$ would imply $y \in D^{2 n+1}\left(V_{1}, G-G_{1}\right)$ contrary to $(2)$.

If $x \in D^{2 n}\left(V_{1}, G-G_{1}\right), y \in D^{2 m-1}\left(W_{1}, G-G_{1}\right)$ then $x \nsucc y$; for $x \succ y$ would imply $y \in D^{2 n+1}\left(V_{1}, G-G_{1}\right)$ contrary to (2).

If $x \in D^{2 m-1}\left(W_{1}, G-G_{1}\right), y \in V_{1}$ then $x \nsucc y$; for $x \succ y$ would imply $y \in$ $D^{2 n}\left(\mathbb{W}_{1}, G-G_{1}\right)$ contrary to $(1)$.

If $x \in D^{2 m-1}\left(W_{1}, G-G_{1}\right), y \in D^{2 n}\left(V_{1}, G-G_{1}\right)$ then $x \nsucc y$; for $x \succ y$ would imply $y \in D^{2 m}\left(W_{1}, G-G_{1}\right)$ contrary to $(2)$.

If $x \in D^{2 m-1}\left(W_{1}, G-G_{1}\right), y \in D^{2 n-1}\left(W_{1}, G-G_{1}\right)$ then $x \nsucc y$; for $x>y$ would imply $y \in D^{2 m}\left(W_{1}, G-G_{1}\right)$ contrary to (2).

(b) Let

$$
W=W_{1} \cup \underset{n=1}{\bigcup} D^{2 n-1}\left(V_{1}, G-G_{1}\right) \cup \underset{m=1}{\bigcup} D^{2 m}\left(W_{1}, G-G_{1}\right)
$$

By ( 3 ),

$$
D=D_{1} \cup S\left(D_{1}, G-G_{1}\right)=V \cup W .
$$

By (1) and (2), $V \cap W=0$. Hence $\mathbb{W}=\mathscr{D}-V$.

If $y \in W_{1}$, then there exists an $x \in V_{1}$ such that $x>y$.

If $y \in D^{2 n-1}\left(V_{1}, G-G_{1}\right)$ then there exists an $x \in V_{1} \cup D^{2 n}\left(V_{1}, G-G_{1}\right)$ such that $x>y$. 
If $y \in D^{2 m}\left(W_{1}, G-G_{1}\right)$, then there exists an $x \in D^{2 m-1}\left(W_{1}, G-G_{1}\right)$ such that $x>y$. This completes the proof.

Corollary. Let $G_{1}$ be a conjunct subgraph of $G, V_{1}$ a solution of $G_{1}$. Suppose that:

(a) no vertex of any $S\left(x_{1}, G-G_{1}\right), x_{1} \in \mathscr{D}_{1}$, is adjacent to any other vertex of $\mathscr{D}_{1}$; and if $x_{1}$ and $x_{1}^{\prime}$ are any two distinct vertices of $\mathscr{D}_{1}$ then

$$
S\left(x_{1}, G-G_{1}\right) \cap S\left(x_{1}^{\prime}, G-G_{1}\right)=0 ;
$$

(b) no

$$
S\left(x_{1}, G-G_{1}\right) \cup\left(x_{1}\right),
$$$$
x_{1} \in D_{1},
$$

contains an unoriented cycle of odd length;

(c) $\oslash-D_{1} \subset S\left(\oslash_{1}, G-G_{1}\right)$.

Then there exists a solution of $G$ which is an extension of $V_{1}$.

Proof. Condition (c) is identical with ( 3 ) of the theorem. We have only to show that (a) and (b) imply ( 1 ) and (2); that is, that if either (1) or (2) were false then $(a)$ or $(b)$ would be violated.

If (1) were false there would exist either

(i) a vertex $x \in D\left(V_{1}, G\right) \cap D^{2 n}\left(V_{1}, G-G_{1}\right)$,

or (ii) a vertex $y \in D\left(V_{1}, G\right) \cap D^{2 n-1}\left(W_{1}, G-G_{1}\right)$,

or (iii) a vertex $z \in V_{1} \cap D^{2 n+1}\left(V_{1}, G-G_{1}\right)$,

or (iv) a vertex $u \in V_{1} \cap D^{2 n}\left(\mathbb{W}_{1}, G-G_{1}\right)$.

In Case (i )

$$
x \in S\left(v_{1}^{i}, G-G_{1}\right) \cap S\left(v_{1}^{j}, G-G_{1}\right)
$$

and by $(\mathrm{a}), i=j$. But then there exists an unoriented cycle of odd length in $S\left(v_{1}^{i}, G-G_{1}\right) \cup\left(v_{1}^{i}\right)$ contrary to (b). In Case (ii), the second part of (a) is contradicted. In Cases (iii) and (iv), the first part of (a) is contradicted. If (2) were false, there would exist either 
(i) a vertex

$$
x \in D^{h}\left(V_{1}, G-G_{1}\right) \cap D^{k}\left(W_{1}, G-G_{1}\right)
$$

for some $h, k$ of the same parity,

or (ii) a vertex

$$
y \in D^{h}\left(v_{1}^{i}, G-G_{1}\right) \cap D^{k}\left(v_{1}^{j}, G-G_{1}\right)
$$

for some $h, k$ of different parities,

or (iii) a vertex

$$
z \in D^{h}\left(w_{1}^{i}, G-G_{1}\right) \cap D^{k}\left(w_{1}^{j}, G-G_{1}\right)
$$

for some $h, k$ of different parities. In Case (i), the second part of (a) is contradicted. In Cases (ii) and (iii), (a) implies $i=j$ and then (b) is contradicted.

Now suppose $G_{0}$ is a nonempty conjunct subgraph of $G_{1}$ and let $V_{0}$ be a solution of $G_{0}$. For each natural number $n$, let $G_{2 n-1}$ be constructed by adjoining to $G_{2 n-2}$ the vertices of $P\left(\oslash_{2 n-2}, G-G_{2 n-2}\right)$, where $\mathscr{D}_{i}=D_{n} G_{i}$, and taking the juncture; that is,

$$
G_{2 n-1}=G_{2 n-2} \cup P\left(\mathscr{D}_{2 n-2}, G-G_{2 n-2}\right) \text {. }
$$

Similarly let

$$
G_{2 n}=G_{2 n-1} \cup S\left(\mathcal{O}_{2 n-1}, G-G_{2 n-1}\right)
$$

Then each $G_{i}$ is a conjunct subgraph of $G_{i+1}$. For $x, y \in \mathscr{D}_{i}, x>y$ relative to $G_{i+1}$ implies $x>y$ relative to $G_{i}$ since at least one endpoint of every arc in $G_{i+1}-G_{i}$ is not in $G_{i}$.

If $G_{0}$ intersects every component of $G$, then

$$
\mathscr{D}=\bigcup_{i=0}^{\infty} \mathscr{D}_{i}
$$

For then every vertex of $G$ is joined to some vertex of $G_{0}$ by a finite unoriented chain and therefore lies in some $G_{i}$. In particular, this is true if $G$ is connected.

THEOREM 12. Let $G_{0}$ be a conjunct subgraph of $G$ which intersects every 


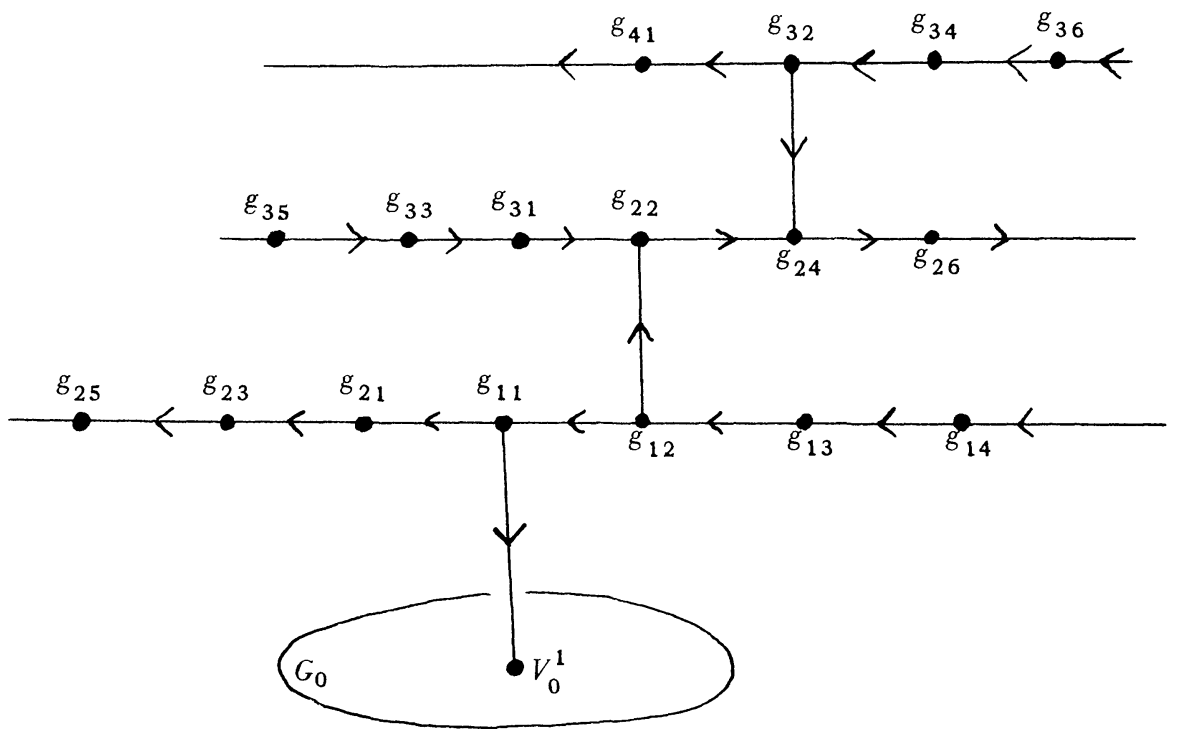

Figure 8 
component of $G$, let $V_{0}$ be a solution of $G_{0}$, and let $G_{i}, i \geq 1$, be defined as above. Suppose that for every even $i, G_{i}$ satisfies Conditions (1), (2), (3) of Theorem 2 relative ${ }^{7}$ to $G_{i+1}$, and that for every odd $i, G_{i}$ satisfies Conditions (1) and (2) of Theorem 11 relative to $G_{i+1}$. Then there exists a solution of $G$ which is an extension of $V_{0}$.

Proof. The solution $V_{0}$ of $G_{0}$ can be extended stepwise to a solution $V_{1}$ of $G_{1}, V_{2}$ of $G_{2}, \ldots, V_{i}$ of $G_{i}, \cdots$ by Theorems 2 and 11 applied alternately. Hence $\cup_{i=0}^{\infty} V_{i}$ is a solution of $G$.

For example, in Figure $8, G_{i}$ has the set of vertices $\mathscr{D}_{i}=\left[g_{i 1}, g_{i 2}, g_{i 3}, \ldots\right]$. Then

$$
\begin{aligned}
V_{1}=\left[g_{12}, g_{14}, \ldots\right] \cup V_{0}, V_{2} & =\left[g_{21}, g_{25}, \cdots ; g_{24}, g_{28}, \ldots\right] \cup V_{1}, \\
V_{3} & =\left[g_{31}, g_{35}, \cdots ; g_{34}, g_{38}, \cdots\right] \cup V_{2}, \\
V_{4} & =\left[g_{41}, \ldots\right] \cup V_{3} .
\end{aligned}
$$

Theorem 11 is a sort of dual to Theorem 2. Theorem 12 merely uses the procedures of Theorems 2 and 11 in alternation. Similar processess dual to those of other preceding theorems can be introduced so as to yield extensions in the direction of successor-sets rather than predecessor-sets, and similar alternating procedures can then be used.

${ }^{7}$ That is, with $G_{i+1}$ in the role of $G$ in Theorem 2 .

\section{REFERENCES}

1. D. König, Theorie der endlichen und unendlichen Graphen, Leipzig, 1936.

2. J. von Neumann and O. Morgenstern, Theory of games and economic behavior, Princeton 1944; 2nd edition 1947.

3. M. Richardson, On weakly ordered systems, Bull. Amer. Math. Soc. 52 (1946), $113-116$.

4. Solutions of irreflexive relations, Ann. of Math. 58 (1953), 573-590.

5. Extension theorems for solutions of irreflexive relations, Proc. Nat. Acad. Sci. 39 (1953), 649-655.

BROOKLYN COLLEGE 


\section{PACIFIC JOURNAL OF MATHEMATICS}

\section{EDITORS}

\section{H.L. ROY DEN}

Stanford University

Stanford, California

E. HEWITT

University of Washington

Seattle 5, Washington

\section{R. P. DILWORTH}

California Institute of Technology

Pasadena 4, California

* Alfred Horn

University of California

Los Angeles 24, California

\section{ASSOCIATE EDITORS}

\begin{abstract}
H. BUSEMANN
HERBERT FEDERER

MARSHALL HALL
\end{abstract}

\section{P.R. HALMOS}

HEINZ HOPF

ALFRED HORN
R.D. JAMES

BØRGE JESSEN

PAUL LÉVY
GEORGE PÓLYA

J.J. STOKER

KOSAKU YOSIDA
UNIVERSITY OF BRITISH COLUMBIA CALIFORNIA INSTITUTE OF TECHNOLOGY UNIVERSITY OF CALIFORNIA, BER KELEY UNIVERSITY OF CALIFORNIA, DAVIS UNIVERSITY OF CALIFORNIA, LOS ANGELES UNIVERSITY OF CALIFORNIA, SANTA BARBARA MONTANA STATE UNIVERSITY

UNIVERSITY OF NEVADA

OREGON STATE COLLEGE

UNIVERSITY OF OREGON
UNIVERSITY OF SOUTHERN CALIFORNIA STANFORD UNIVERSITY UNIVERSITY OF UTAH WASHINGTON STATE COLLEGE UNIVERSITY OF WASHINGTON

AMERICAN MATHEMATICAL SOCIETY HUGHES AIRCRAFT COMPANY SHELL DEVELOPMENT COMPANY

Mathematical papers intended for publication in the Pacific Journal of Mathematics should be typewritten (double spaced), and the author should keep a complete copy. Manuscripts may be sent to any of the editors. Manuscripts intended for the outgoing editors should be sent to their successors. All other communications to the editors should be addressed to the managing editor, Alfred Horn, at the University of California Los Angeles 24, California.

50 reprints of each article are furnished free of charge; additional copies may be obtained at cost in multiples of 50 .

The Pacific Journal of Mathematics is published quarterly, in March, June, September, and December. The price per volume (4 numbers) is $\$ 12.00$; single issues, $\$ 3.50$; back numbers (Volumes $1,2,3$ ) are available at $\$ 2.50$ per copy. Special price to individual faculty members of supporting institutions and to individual members of the American Mathematical Society: $\$ 4.00$ per volume; single issues, $\$ 1.25$.

Subscriptions, orders for back numbers, and changes of address should be sent to the publishers, University of California Press, Berkeley 4, California.

Printed at Ann Arbor, Michigan. Entered as second class matter at the Post Office, Berkeley, California.

* During the absence of E.G. Straus.

UNIVERSITY OF CALIFORNIA PRESS - BERKELEY AND LOS ANGELES 


\section{Pacific Journal of Mathematics}

\section{Vol. 5, No. $4 \quad$ December, 1955}

Richard Horace Battin, Note on the "Evaluation of an integral occurring in servomechanism theory" ............................. 481

Frank Herbert Brownell, III, An extension of Weyl's asymptotic law for eigenvalues................................. 483

Wilbur Eugene Deskins, On the homomorphisms of an algebra onto Frobenius algebras .................................. 501

James Michael Gardner Fell, The measure ring for a cube of arbitrary dimension ....................................... 513

Harley M. Flanders, The norm function of an algebraic field extension. II ............................................ 519

Dieter Gaier, On the change of index for summable series ............ 529

Marshall Hall and Lowell J. Paige, Complete mappings of finite groups . . . . 541

Moses Richardson, Relativization and extension of solutions of irreflexive relations..................................... 551

Peter Scherk, An inequality for sets of integers .................. 585

W. R. Scott, On infinite groups ........................... 589

A. Seidenberg, On homogeneous linear differential equations with arbitrary constant coefficients ......................................... 599

Victor Lenard Shapiro, Cantor-type uniqueness of multiple trigonometric integrals...

Leonard Tornheim, Minimal basis and inessential discriminant divisors for a cubic field...

Helmut Wielandt, On eigenvalues of sums of normal matrices ... 